\title{
Motion direction tuning in human visual cortex
}

\author{
Manuel Mercier, ${ }^{1}$ Sophie Schwartz, ${ }^{2}$ Christoph M. Michel ${ }^{3}$ and Olaf Blanke ${ }^{1,4}$ \\ ${ }^{1}$ Laboratory of Cognitive Neuroscience, Brain Mind Institute, EPFL, Lausanne, Switzerland \\ ${ }^{2}$ Laboratory for Neurology and Imaging of Cognition, Department of Neuroscience, University of Geneva, Geneva, Switzerland \\ ${ }^{3}$ Functional Brain Mapping Laboratory, University Hospital, Geneva, Switzerland \\ ${ }^{4}$ Department of Neurology, University Hospital, Geneva, Switzerland
}

Keywords: direction, EEG, human, motion, vision

\begin{abstract}
A number of electrophysiological studies have been conducted in recent years in order to clarify the dynamics of visual motion processing in the human brain. Using a variety of event-related potential (ERP) measures, several parameters such as onset, offset, contrast and velocity have been investigated, while a critical aspect of visual motion, that of direction, has received less attention. Here we used multichannel electroencephalography and distributed source localization to study brain activity for different directions of visual motion using random dot stimuli. Our data reveal differential extrastriate activation at 164-226 ms after motion onset that coded for motion direction with different ERP maps and underlying electrical generators for each tested direction. This activation was paralleled initially (164-186 ms) by a distinct extrastriate activation encoding whether the motion stimulus consisted of directed motion stimuli (as above) or contained undirected incoherent motion (control stimulus). Application of a linear inverse solution localized the brain activity for each tested motion direction to distinct brain regions within the same larger network of extrastriate brain regions. These regions included bilateral temporo-occipital and bilateral parieto-occipital cortex. The present data in healthy subjects are compatible with extrastriate activity that is tuned to different directions of visual motion. This extends previous clinical data and suggests the presence of distributed macroscopic motion direction tuning in primate extrastriate cortex that may complement the classical microscopic motion tuning at the columnar level.
\end{abstract}

\section{Introduction}

The visual environment of most animals is highly dynamical, and the detection and interpretation of motion patterns is crucial for predicting the future state of one's dynamic world. The speed and direction of such moving stimuli are crucial variables, and many studies have investigated the neural underpinnings of visual motion processing in human and non-human primates. Towards that end, single-unit electrophysiology in monkeys (Albright \& Stoner, 1995), neuroimaging in humans, such as functional magnetic resonance imaging (Culham et al., 2001), and event-related potentials (ERPs; see below) have been used.

Concerning the electrophysiological dynamics of visual motion processing in humans, ERP studies consistently have revealed three main ERP components evoked during visual motion processing: the P120, N170 and P220 component. For the P120 it was shown that its amplitude and latency is influenced by pattern reversal, onset and offset, as well as by changes in luminance and contrast of the motion stimulus (Kubova \& Kuba, 1993; Bach \& Ullrich, 1997; Kuba et al., 2007). Using magnetoencephalography (MEG) and electrical source reconstruction, Nakamura \& Ohtsuka (1999) localized the generator of

Correspondence: Dr O. Blanke, ${ }^{1}$ Laboratory of Cognitive Neuroscience, as above. E-mail: olaf.blanke@epfl.ch

Received 11 April 2008, revised 7 November 2008, accepted 14 November 2008 the P120 in the primary visual cortex. The N170 component is assumed to be the most important in visual motion processing as measured by ERPs. Thus, the amplitude and latency of the N170 has been proposed to reflect the velocity of the motion stimulus (Muller et al., 1998; Bundo et al., 2000; Maruyama et al., 2002), and its amplitude is modulated by the global coherence of the motion signal (Niedeggen \& Wist, 1998, 1999; Nakamura et al., 2003). Electroencephalography (EEG) and MEG studies have localized the N170 in occipito-temporal or occipito-parietal cortex (Probst et al., 1993; Nakamura \& Ohtsuka, 1999), but also at more posterior sites (estimated in close proximity to area V3a; Nakamura \& Ohtsuka, 1999; Schellart et al., 2004). Using MEG and functional magnetic resonance imaging (fMRI), Ahlfors et al. (1999) and Bundo et al. (2000) localized the N170 to a network of brain areas, including middle temporal area MT+/V5, V3a, occipital cortex, the posterior superior temporal cortex and frontal eyes field. The amplitude of the P220 is also modulated by stimulus velocity (Hoffmann \& Bach, 1997), but has only rarely been investigated (compared with both earlier motion components). Yet, several studies suggest its involvement in the processing of complex visual motion stimuli. This evidence consists of observations that its amplitude increases with motion complexity (such as contracting or expanding radial motion; Kuba et al., 2007).

In the present study, we used ERP trace analysis, topographical analysis and distributed source localization techniques to investigate 
the timing and location of brain activity during the processing of motion directions. Motivated by recent data suggesting a differential implication of extrastriate cortex in different motion directions as investigated by cortical stimulation in epileptic patients (Blanke et al., 2002) and focal brain damage in neurological patients (Blanke et al., 2003), we here investigated electrophysiological correlates of motion direction in healthy human observers.

\section{Materials and methods}

\section{Subjects}

Ten healthy volunteers (mean age $\pm \mathrm{SD}, 26.4 \pm 3.7$ years; four female) participated in the study. Written informed consent was provided to participate in the experiment, which was approved by the Ethical Committee of the University Hospital of Geneva (in accordance with the Declaration of Helsinki). All subjects were righthanded, had no neurological or psychiatric illness, and had normal or corrected-to-normal vision.

\section{Stimuli and procedure}

Stimuli were black and white random dot kinematograms (RDK), as used in previous studies examining direction of motion perception in brain-damaged and epileptic patients (Blanke et al., 2002, 2003, 2007). RDKs were presented within a square of $12 \times 12^{\circ}$ in the central visual field. Viewing distance was $120 \mathrm{~cm}$. We used a 20-inch monitor (Sony Trinitron Multiscan model no. GDM-20SE1VT; $640 \times 480$ pixels resolution), with $32.5 \mathrm{~cd} / \mathrm{m}^{3}$ as mean luminance for white. Stimuli were presented with a $70-\mathrm{Hz}$ frame rate. Each random dot field contained 1000 dots (each dot had a diameter of $0.68^{\circ}$ ), randomly positioned to maintain constant average density across the presentation field (the same amount of dots was present at the four boundaries). In all conditions individual dots were assigned a random lifetime (15-500 ms). As a result, dots could appear or vanish at any position within the $12 \times 12^{\circ}$ square (as dot density was constant, the RDK did not lead to any perceptual differences at the boundaries). The velocity of dot displacement was $2 \%$ during each presentation. RDK were presented for durations of $500 \mathrm{~ms}$, and alternated with a white fixation cross displayed on a black background and presented for $700 \mathrm{~ms}$.

Four cardinal directions (all dots moved rightward, leftward, upward or downward) were tested. On each trial, the direction of the RDK stimulus was randomly selected. An additional incoherent motion condition was also included as a control condition. The total number of repetitions for each stimulus condition was 80. Stimuli were produced using the Cogent Graphics Matlab toolbox, and displayed using E-prime (Psychology Software Inc., Pittsburgh, PA, USA).

Subjects performed a motion direction detection task, and were asked to indicate by a button press whether they perceived directed coherent motion or not. Button press responses were counterbalanced between subjects (half of the subjects responded with the index finger for directed motion and with the middle finger for incoherent motion; the other half responded inversely).

\section{EEG acquisition and preprocessing}

Continuous EEG was acquired in a Faraday cage, with a Geodesics Netamps system (Electrical Geodesics, Eugene, Oregon, USA), from 128 electrodes (impedances $<50 \mathrm{k} \Omega$; vertex reference; $500 \mathrm{~Hz}$ sampling rate; band pass filter $0.1-200 \mathrm{~Hz}$ ). EEG epochs were calculated from $100 \mathrm{~ms}$ before to $300 \mathrm{~ms}$ after stimulus onset, band pass filtered between 1 and $30 \mathrm{~Hz}$, and averaged separately for each of the five stimulus conditions and for each subject to calculate the ERP. In addition to the application of an automated artefact rejection $( \pm 100 \mu \mathrm{V})$, all accepted trials were visually inspected at the level of traces, and scalp topography for a window size of -200 to $+700 \mathrm{~ms}$, to reject epochs with transient contaminating noise like eye blinks, eye movements and muscular artefacts.

Baseline was defined as the mean value of the averaged amplitude from $100 \mathrm{~ms}$ before to $30 \mathrm{~ms}$ after stimulus onset (Hoffmann et al., 2001).

For each subject's ERP, artefacted electrodes were interpolated using the 3D spline method. ERP data were down-sampled to a 111-channel montage used in the estimation of the inverse solution. ERPs were recalculated to a frontal reference for optimizing visual EP amplitudes (Ffytche et al., 1995; Bach \& Ullrich, 1997; Hoffmann et al., 1999). Group averaging was calculated with ERPs normalized to their mean global field power (corresponds to the spatial standard deviation of the scalp electric field at a certain point in time).

\section{Trace analysis: amplitude and latency}

For the P120 component we analysed traces recorded at Oz, and for the P220 component at Cz, as done by Hoffmann et al. (2001) and Hoffmann \& Bach (2002). For the N170 component, we selected for each subject the $\mathrm{Ot}^{*}$ derivation, introduced by Hoffmann et al. (1999). Those authors observed that the N170 component is often lateralized and constant within but not across subjects, and proposed to use for each subject the $\mathrm{O} 1$ or $\mathrm{O} 2$ potential with maximal N170 amplitude. This was labelled Ot*. Accordingly, we determined for each subject the $\mathrm{Ot}^{*}$ value and used it further for statistical analysis (Bach \& Hoffmann, 2000; Hoffmann et al., 2001; Maurer \& Bach, 2003; Heinrich et al., 2004, 2005).

Motion direction comparison, peak latency and amplitude for the three components were analysed with repeated-measures ANOVA, and with additional planned Fisher-LSD post hoc comparisons. Paired $t$-tests were applied for comparisons between directed coherent motion vs. incoherent motion. For each individual subject and condition, peak amplitude and latency were extracted. Figure 2A shows traces corresponding to each component and electrode location for the four motion directions (see Fig. 3B for incoherent motion). The P120 component (at electrode $\mathrm{Oz}$ ) peaked at $144 \pm 2.1 \mathrm{~ms}$ with $8.96 \pm 1.52 \mu \mathrm{V}$ amplitude. The N170 component $\left(\mathrm{Ot}^{*}\right)$ peaked at $179 \pm 3.2 \mathrm{~ms}$ with a negative amplitude of $1.09 \pm 1 \mu \mathrm{V}$. The P220 component (at electrode $\mathrm{Cz}$ ) peaked at $212 \pm 3.1 \mathrm{~ms}$ with an amplitude of $3.37 \pm 0.9 \mu \mathrm{V}$ (all data $\pm \mathrm{SD}$ ).

\section{ERP mapping: topographic pattern analysis}

Recording activity from many different sites simultaneously over the scalp allows the construction of topographic maps evolving over time. This can be used to determine when map configurations change and/or differ between experimental conditions relying on the assumption that whenever the spatial configuration of the electric field on the scalp differs, different neuronal populations are active in the brain. For this we used a spatio-temporal segmentation algorithm derived from spatial cluster analysis, as described previously (Lehmann et al., 1987; Pascual-Marqui et al., 1995; Michel et al., 1999, 2001; Murray et al., 2004, 2008; Blanke et al., 2005; Arzy et al., 2006; Thierry et al., 2007). The method applies a modified k-means cluster analysis for identifying the most dominant scalp topographies appearing in the group-averaged ERPs of each condition 
over time. This spatial pattern analysis summarizes the whole ERP data by a limited number of scalp potential configurations. The optimal number of segmentation maps that explains the whole data set is determined by a modified cross-validation criterion optimizing the degrees of freedom and the explained variance (Pascual-Marqui et al., 1995).

Even though such cluster analysis per se does not rely on any temporal characteristics of the data, it leads to a segmentation of the ERPs into discrete successive time periods. This is due to the fact that map topographies do not randomly change over time but remain stable in a certain configuration for certain periods and then change to a new stable spatial configuration. It has been proposed that each of these 'segmentation maps' represents a given 'functional microstate' of the brain or a given computational step during information processing.

The dominant scalp topographies (identified in the group-averaged data) were then fitted to the ERPs of each individual subject using spatial fitting procedures. For each time point of the individual subject's ERP, the scalp topography was compared by means of normalized spatial correlation to each segmentation map, and was labeled according to the one with which it correlated best. From this fitting procedure, we determined the global explained variance (GEV; corresponding to the goodness of fit of a map during a certain time period) and the duration that a given segmentation map was observed for a given condition across subjects (frequency of occurrence). Thus, we measured those values of the grand average maps not only for their corresponding individual data (same condition), but also for individual data of the other condition. Values were then subjected to statistical analysis using repeated-measure ANOVA with maps and conditions as main factors, and individual GEV and duration as repeated-measures.

For motion direction comparison, we separately analysed, from 0 to $300 \mathrm{~ms}$ after stimulus onset, all six possible direction pairs corresponding to each direction comparison: right vs. left; right vs. up; right vs. down; left vs. up; left vs. down; and up vs. down. This procedure tests whether certain maps can distinguish one direction from all the other directions. Using the fitting method described above, such direction-specific maps (that dissociated between conditions) were searched for within individual subject data. A repeated-measures ANOVA using stimulus condition (two directions of motion) and segmentation maps as within-subject factors was then conducted on measures of map GEV and map duration for each of the six pairs of possible comparisons.

\section{Vertical Movement}

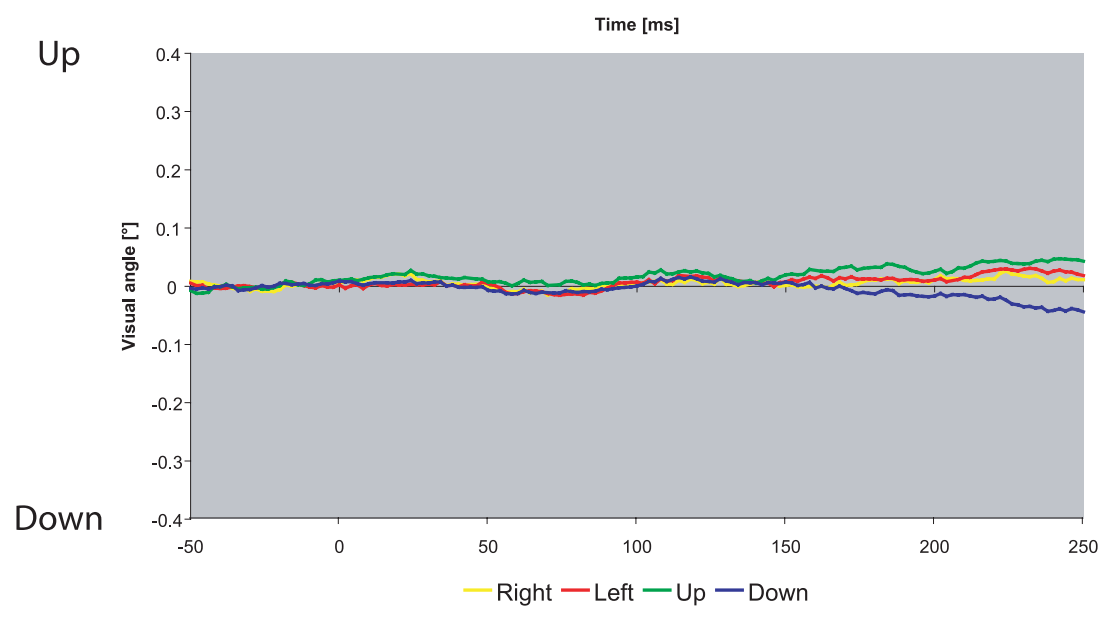

Horizontal Movement

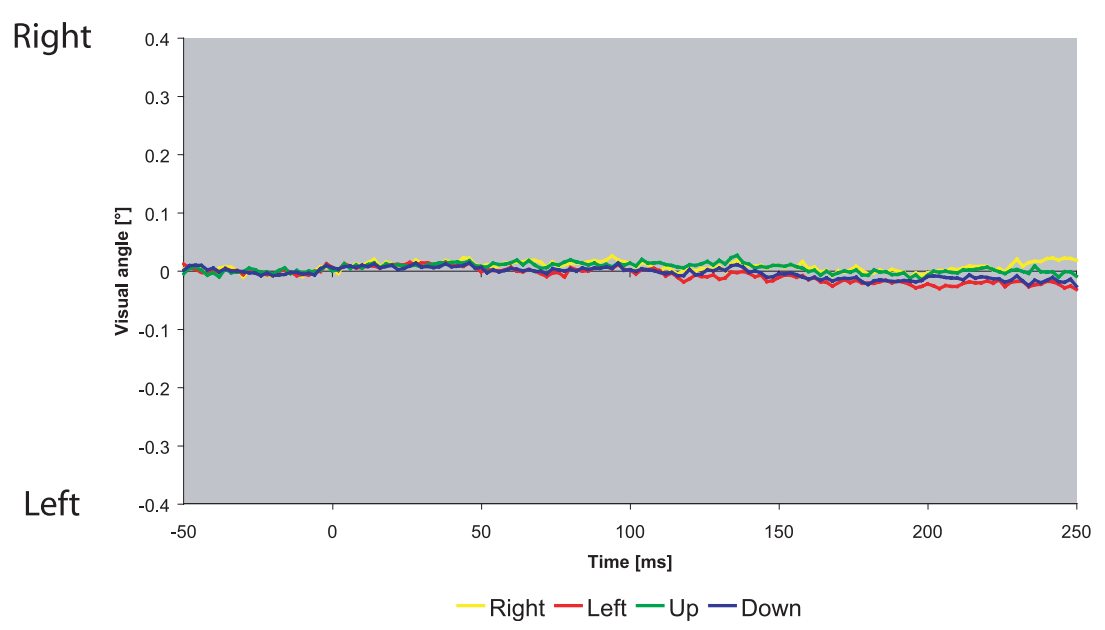

FIG. 1. Eye movements. Averaged eye movements recorded during the directional conditions using video-infrared recording. $0 \% \mathrm{CM}$ have been subtracted from each direction of $100 \%$ CM. Motion in the right (yellow), left (red), up (green) and down directions (blue) are indicated separately. The top figure shows vertical eye movements, and the bottom figure shows horizontal eye movements. 
Next we analysed whether incoherent motion differed from directed coherent motion. A repeated-measures ANOVA using stimulus condition (incoherent motion vs. directed motion) and segmentation map as within-subject factors was then conducted, again using the GEV of the segmentation maps as well as map duration.

In order to ensure that such potential dissociations are specific for a certain time period corresponding to one segmentation map, we also tested whether maps preceding and following any map of interest showed such differences between conditions. However, as we consistently found the same maps for different conditions during these 'border' periods, we could not test for differences in map duration, but only for goodness of fit assessed by GEV.

\section{Distributed source localization}

Brain generators were estimated using a distributed linear inverse solution on local auto-regressive average (LAURA; Grave de Peralta Menendez et al., 2001, 2004). This method uses a realistic head model with 4024 solutions points equally distributed within the grey matter of the Montreal Neurological Institute's average brain. This method deals with multiple simultaneously active sources of a priori unknown location, and makes no assumptions regarding the number or location of active sources. This linear-distributed inverse solution selects the source configuration that best mimics the biophysical behaviour of electrical field and produces a unique estimation of the current source's density inside the brain. That is, the estimated activity at one point depends on the activity at neighbouring points as described by electromagnetic laws (Grave de Peralta-Menendez \& GonzalezAndino, 1998).

Source reconstruction was performed at two levels. First, we applied LAURA to the template maps and thus over the time periods showing topographical differences for the different motion directions as well as coherent vs. incoherent motion. This was done in order to visualize the brain generators for the different conditions as identified by the segmentation procedure and statistical topographical analysis described above.

Second, we performed statistical analysis at the level of the source reconstruction. For this, we determined for each participant and each

A
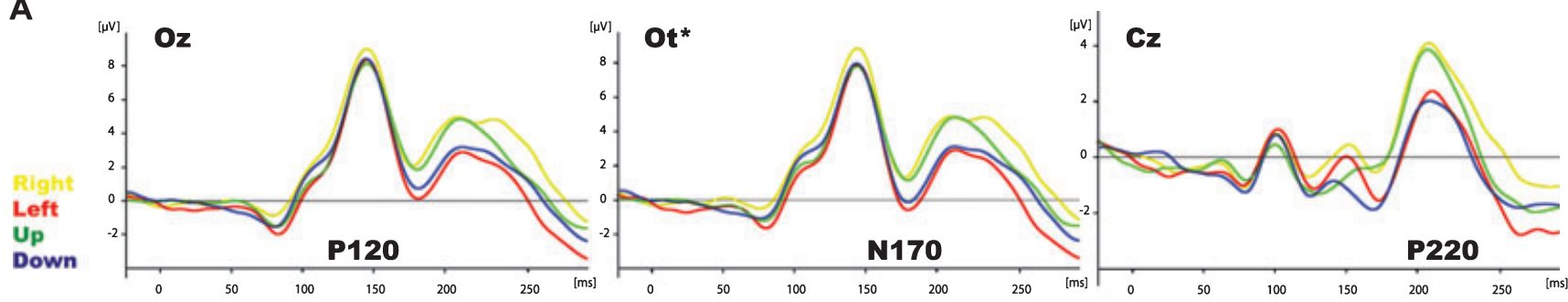

B
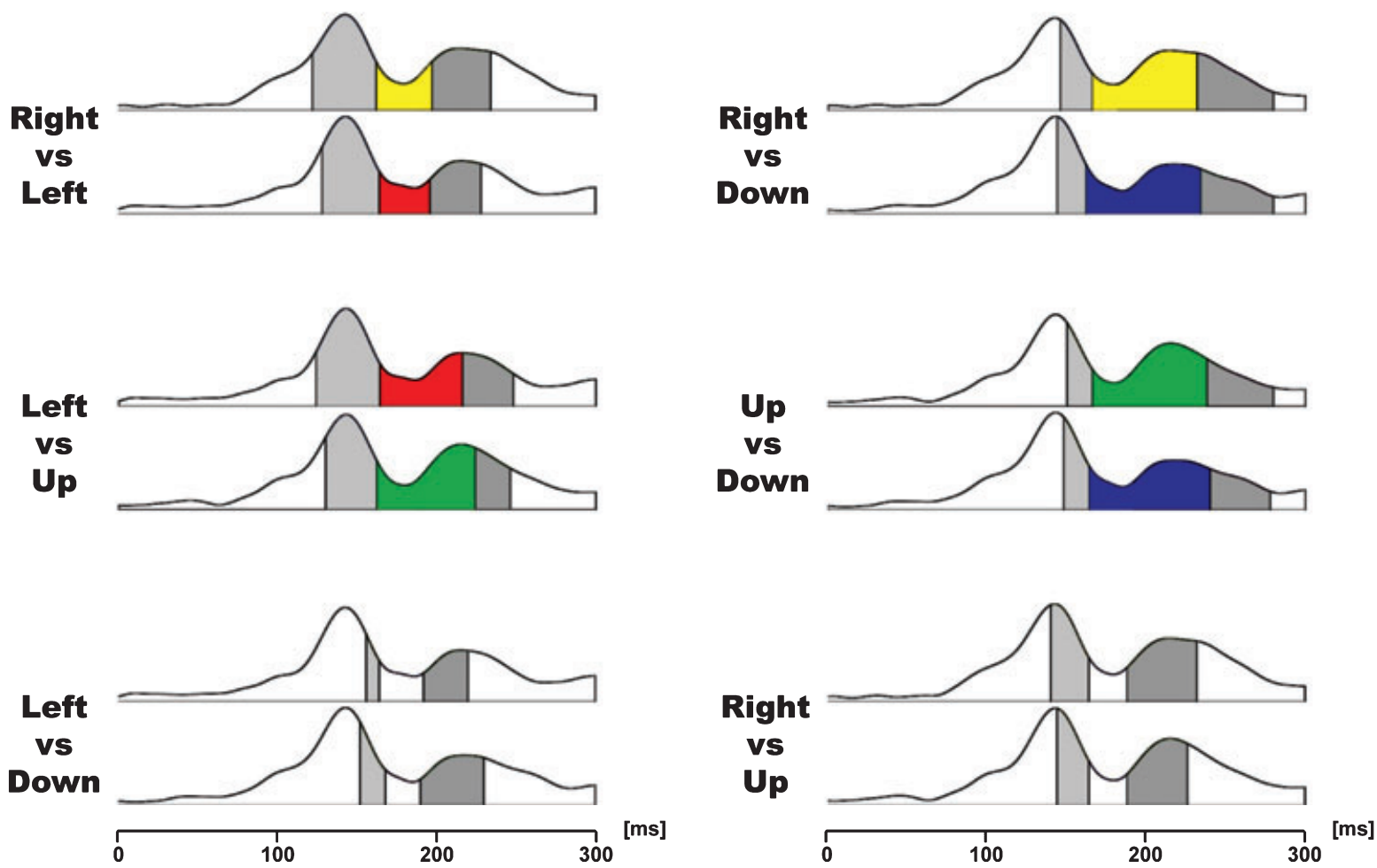

FIG. 2. Directional tuning as revealed by ERP trace analysis and ERP mapping. (A) ERP traces corresponding to the four directed motions are plotted. Directed motion towards the right (yellow), left (red), up (green) and down (blue) are shown. Respectively from left to right are plotted the P120 component at Oz electrodes, the N170 component at Ot* and the P220 component at Cz. (B) Topographical clustering results for each directional motion pair (same colour as in A). Only right vs. up and left vs. down comparisons did not reveal any distinct map between 164 and $226 \mathrm{~ms}$. 
directional condition (and the incoherent motion condition) the mean corresponding EP map during the time period suggested by the topographical analysis [directional (coherent) conditions: 164$226 \mathrm{~ms}$; incoherent motion: 164-186 ms]. LAURA was applied to each individual EP map for each directional condition (and the incoherent motion condition). These inverse solutions were subjected to statistical analysis (pairwise $t$-test for each solution point), with subjects as repeated-measure and stimulus condition as within-subject factor.

\section{Eye movement recordings}

Electroocculography (EOG)

EOG was computed using a bipolar montage with electrodes positioned on the left and right side of the eyes (for horizontal eye movements) as well as above and below the eyes (for vertical movements). The same parameters for filtering, baseline correction and averaging were applied as described above. For statistical analysis we used time-point paired $t$-tests of the eye movement traces for each directional condition, with the control condition (incoherent motion) from 0 to $300 \mathrm{~ms}$ post-stimulus revealing the absence of significant eye movements as detectable by this technique.

\section{Video-based tracking}

In eight additional participants (mean age $27.7 \pm 3.7$ years; three female; all with normal or corrected vision; all right-handed) we measured eye position using a video-based tracking system (SMI eye tracker - Iview $\mathrm{X}^{\mathrm{TM}}$ Hi-Speed, Teltow/Berlin, Germany; sampling rate: $500 \mathrm{~Hz}$, tracking resolution: $<0.01^{\circ}$ ) during the four directional conditions and the incoherent motion condition. The eye position from both eyes was averaged. The mean eye signal for each direction across all trials was then calculated. This was done time-locked to stimulus onset for the period from -200 to $400 \mathrm{~ms}$ (baseline corrected from -50 to $0 \mathrm{~ms}$ ). This analysis revealed correct fixation in all conditions, and excluded eye movements that might have artefacted our EEG data during the appearance of the directional EP maps (no horizontal or vertical eye movement signals $>0.1^{\circ}$ of visual angle were observed). Our analysis revealed smooth pursuit eye movements with very small amplitudes of $0.1^{\circ}$ (or smaller) of visual angle (our visual stimuli extended $12 \times 12^{\circ}$ of visual angle) starting at $250 \mathrm{~ms}$ after stimulus onset (Fig. 1) and thus after the occurrence of the directional EP maps (see below).

\section{Results}

\section{Behavioural results}

The response accuracy was $90.9 \%$ (SD: $4.6 \%$ ) and reaction time $270 \mathrm{~ms}$ (SD, $20 \mathrm{~ms}$ ). A repeated-measures ANOvA using stimulus condition as within-subject factors was conducted, both on accuracy and reaction time. This statistical analysis did not reveal any significant differences between condition, neither for accuracy nor reaction time ( $P>0.69$ for both parameters).

\section{Trace analysis}

\section{Motion directions: amplitude}

Whereas our analysis did not reveal a significant direction effect for the amplitude of the P120 component $\left(F_{3,27}=0.68 ; P=0.57\right)$, this was the case for the amplitude of the N170 component $\left(F_{3,27}=3.21\right.$;
TABLE 1. Single-trace analysis: comparing the amplitudes of the N170 and P220 waves

\begin{tabular}{|c|c|c|}
\hline \multirow[b]{2}{*}{ Conditions being compared } & \multicolumn{2}{|l|}{$P$-value } \\
\hline & N170 & $\mathrm{P} 220$ \\
\hline Right vs. left & $<0.01$ & $<0.05$ \\
\hline Right vs. up & $=0.48$ & $=0.91$ \\
\hline Right vs. down & $=0.053$ & $<0.05$ \\
\hline Left vs. up & $<0.05$ & $<0.05$ \\
\hline Left vs. down & $=0.43$ & $=0.77$ \\
\hline Up vs. down & $=0.20$ & $<0.05$ \\
\hline
\end{tabular}

$P=0.039$ ). Post hoc LSD-Fisher tests for the N170 (paired comparisons) showed that the effect was due to significant differences between right vs. left, and left vs. up (a trend was found for right vs. down; Table 1). We note that the $\mathrm{Ot}^{*}$ derivation was selected six times at $\mathrm{O} 1$ and four times at $\mathrm{O} 2$. This was also reflected in the mean topographical maps corresponding to this component, showing slightly larger amplitude over left than right posterior scalp regions. A significant direction effect was also found for the amplitude of the P220 component $\left(F_{3,27}=4.31 ; P=0.013\right)$. Post hoc analysis revealed differences between the following directions: right vs. left; right vs. down; left vs. up; and up vs. down (see Table 1 and Fig. 2A).

\section{Motion directions: latency}

With respect to latency at these same electrode locations, no directional effects were found for any of the three components (P120 component: $\quad F_{3,27}=1.46 ; \quad P=0.25 ; \quad$ N170 component: $F_{3,27}=0.55 ; P=0.65 ;$ P220 component: $F_{3,27}=1.27 ; P=0.30$ ).

\section{Directed motion vs. incoherent motion: amplitude}

Statistical comparison of directed coherent motion vs. incoherent motion found no significant direction effect for the P120 $\left(t_{9}=1.08\right.$; $P=0.31)$ or the $\mathrm{P} 220$ component $\left(t_{9}=1.19 ; P=0.27\right)$. A significant direction effect due to a larger amplitude for incoherent motion was observed for the N170 component $\left(t_{9}=2.93 ; P=0.017\right.$; see Fig. $\left.3 \mathrm{~A}\right)$.

\section{Directed motion vs. incoherent motion: latency}

No significant direction effects were found for the P120 $\left(t_{9}=2.17\right.$; $P=0.06)$, for the N170 $\left(t_{9}=0.73 ; P=0.48\right)$ or the P220 component $\left(t_{9}=2.11 ; P=0.06\right)$ between directed coherent motion vs. incoherent motion.

\section{ERP mapping: topographic pattern (map) analysis}

\section{Motion directions}

For the period from stimulus onset to $300 \mathrm{~ms}$, map analysis revealed topographical differences between the following motion directions: right vs. left; right vs. down; left vs. up; up vs. down. For all comparisons these differences were found during the time period from 164 to $226 \mathrm{~ms}$. The two other direction comparisons (right vs. up and left vs. down) did not show any map differences (Fig. 2B). This was confirmed by statistical analysis for map duration and GEV (Table 2). These directional differences (interaction between maps and conditions) were selective for this time period, and we found no statistical differences for the maps occurring before and after the 164 to 226-ms time period. In addition we note that the time period suggested by topographical analysis overlaps with the time period evaluated by the N170 and the P220 components. 
TABLE 2. Topographical analysis of six directional EP map comparisons*

\begin{tabular}{lll}
\hline & \multicolumn{2}{l}{$P$-value } \\
\cline { 2 - 3 } Conditions being compared & Duration & GEV \\
\hline Right vs. left & $<0.01$ & $<0.05$ \\
Right vs. down & $<0.005$ & $=0.001$ \\
Left vs. up & $<0.005$ & $<0.05$ \\
Up vs. down & $=0.056$ & $<0.01$ \\
Right vs. up & - & $=0.44$ \\
Left vs. down & - & $=0.31$ \\
\hline
\end{tabular}

*The six directional EP map comparisons were carried out between all pairs of motion direction between 164 and 226 ms. GEV, global explained variance.

\section{Directed motion vs. incoherent motion}

Map analysis between directed and incoherent motion also revealed topographical differences in the time period from 164 to $186 \mathrm{~ms}$. Figure 3B shows the presence of a specific map for directed and incoherent motion during this period, suggesting that one map reflects brain activity during the processing of incoherent motion (black map in Fig. 3B) and one map during directed motion (grey map in Fig. 3B). This was confirmed by statistical analysis for map duration and GEV (significant interaction for duration: $t_{9}=7.42 ; P=0.023$; and for GEV: $t_{9}=9.55 ; P=0.013$ ), with factors map and condition (see Fig. 3B). Both maps did not differ in duration or GEV before or after this time period. To further confirm that this effect is specific for the time period of 164-186 ms, we ran an additional statistical analysis on the GEV and duration of these maps before and after this time period, as well as on the GEV of maps occurring before and after that period. These were not found to be significant.

\section{Inverse solution}

\section{Motion directions}

Source reconstructions were computed for the maps characteristic to each direction, as identified by the segmentation procedure (Fig. 4). For right motion the brain activation was centred in the left temporooccipital cortex (maximum; Talairach coordinates: $X=-36, Y=-75$, $Z=-12)$ and right temporo-occipital cortex $(X=48, Y=-58$, $Z=-11)$. Less strong activations were found in the right parietooccipital cortex $(X=24, Y=-72, Z=42)$ and right occipital cortex $(X=18, Y=87, Z=-3)$. Left motion showed strongest activation in the right temporo-occipital cortex $(X=48, Y=-58, Z=-6)$ and weaker activations in the left temporo-occipital $(X=-36, Y=-75$, $Z=-12)$, left parieto-temporo-occipital $(X=-48, Y=-60, Z=26$ ), right occipital $(X=18, Y=-87, Z=-3)$ and right parieto-occipital cortex $(X=18, Y=-78, Z=37)$. Up motion activated left (maximum; $X=-36, Y=-75, Z=-12$ ) and right temporo-occipital cortex $(X=48, Y=-58, Z=-11)$, as well as right occipital $(X=18$, $Y=-87, Z=-3$ ) and bilateral parieto-occipital cortex (right: $X=18$, $Y=-78, Z=37$; left: $X=-18, Y=-77, Z=31$ ). Down motion showed strongest activation in the right parieto-occipital cortex $(X=24, \quad Y=-72, Z=42)$ and weaker activities in the right temporo-occipital cortex $(X=48, Y=-58, Z=-6)$ and left parietooccipital cortex $(X=-18, Y=-77, Z=31)$. Peaks of activation in the left temporo-occipital cortex were therefore more posterior and less lateral than those in the right temporo-occipital cortex: a finding that is compatible with previous neuroimaging data (Sunaert et al., 1999; Dumoulin et al., 2000). Although globally an extended cortical network in the temporo-occipital and parieto-occipital cortex was activated, the main peak of activation for the different tested directions was localized in the temporo-occipital cortex for three directions (only down motion was localized in the parieto-occipital cortex). Strongest activation in the temporo-occipital cortex was found either in the left hemisphere (right, up) or right hemisphere (left).

These differences were confirmed by statistical analyses at the level of the inverse solutions. For the four directional comparisons that revealed differences in the topographical analysis we also found significant differences (all $P<0.01$ - uncorrected) in the abovementioned areas (Fig. 5). Comparison of right vs. left revealed significant differences in the right temporo-occipital cortex $(X=47$, $Y=-52, Z=-5)$ and right parieto-occipital cortex $(X=5, Y=-50$, $Z=48$ and $X=47, Y=-43, Z=53)$. Right vs. down comparison showed differences in the right $(X=65 ; Y=-28 ; Z=19$ and $X=35$; $Y=-58 ; Z=-5)$ and left $(X=-35 ; Y=-81 ; Z=5)$ temporooccipital cortex. Left vs. up comparison led to significant differences in the right parieto-occipital cortex $(X=11, Y=-62, Z=27)$ and left temporo-occipital cortex $(X=-59 ; Y=-46 ; Z=9)$. Comparisons of up vs. down revealed significant differences in the left and right parieto-occipital cortex (respectively: $X=-41, Y=-81, Z=11$ and $X=29 ; Y=-80 ; Z=16)$ and left parieto-occipital cortex $(X=-29$; $Y=-49 ; Z=65)$. No statistical differences were found for right vs. up and left vs. down comparisons. Applying Bonferroni correction (number of scalp electrodes) at the level of the inverse solution revealed significant differences in extrastriate cortex in two directional comparisons and in two regions, in the right temporo-occipital cortex (right vs. left) and in the left parieto-occipital cortex (up vs. down).

\section{Incoherent motion}

For incoherent motion (Fig. 6A), maximal activation arose in the left parieto-occipital $(X=-18, Y=-77, Z=26)$, whilst other activities were found in the right parieto-occipital $(X=18, Y=-77, Z=31)$, and left and right temporo-occipital cortex $(X=-48, Y=-58$, $Z=-6 ; X=48, Y=-58, Z=-6$, respectively). This was again confirmed by statistical comparison at the level of the inverse solution (Fig. 6B). Significant differences in the left temporo-occipital cortex $(X=-47, Y=-64, Z=-1)$, left parieto-occipital cortex $(X=-23$, $Y=-93, Z=8)$ and left temporal cortex $(X=-59, Y=-40, Z=-5)$ were observed.

\section{Discussion}

Based on single-trace analysis, ERP mapping and distributed source localization, our data show that brain activity in the extrastriate cortex differentiates between directed motion and incoherent random motion (motion effect) and between different motion directions (directional effect). Moreover, the timing of both mechanisms differed: the motion effect was observed in the time period from 164 to $186 \mathrm{~ms}$ after the onset of a visual motion stimulus, whereas the directional effect also started at $164 \mathrm{~ms}$ but lasted longer, until $226 \mathrm{~ms}$.

\section{Single-trace analysis}

Both effects were reflected at the level of ERP components of single scalp electrodes. The directional and motion effect were observed for the N170 component. Previous studies investigating visual motion perception showed that the amplitude of the N170 component reflects the velocity (Muller et al., 1998; Maruyama et al., 2002) and the signal strength of the motion signal (Niedeggen \& Wist, 1998, 1999; Nakamura et al., 2003). Moreover, it has been shown that the N170 component at occipito-temporal electrodes (i.e. Ot*) adapts to motion 
direction (Bach \& Ullrich, 1994; Muller et al., 1998; Hoffmann et al., 1999, 2001; Bach \& Hoffmann, 2000; Heinrich et al., 2004). Thus, Hoffmann et al. (1999) showed that for a given motion direction (presented during the adaptation phase) the amplitude of the N170 component to a subsequently presented motion stimulus is smaller for the adapted direction than for any other direction (but especially the
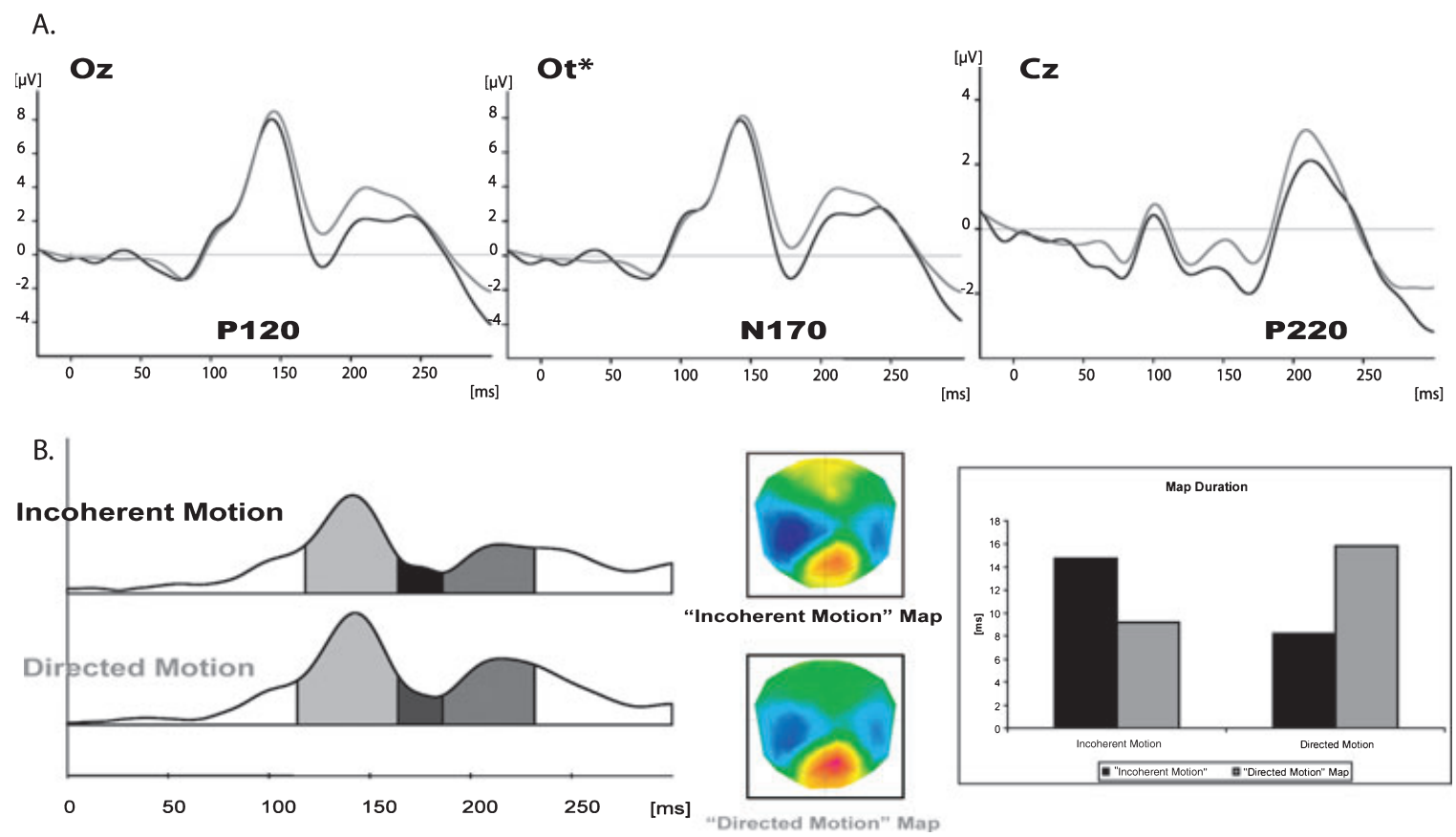

FIG. 3.
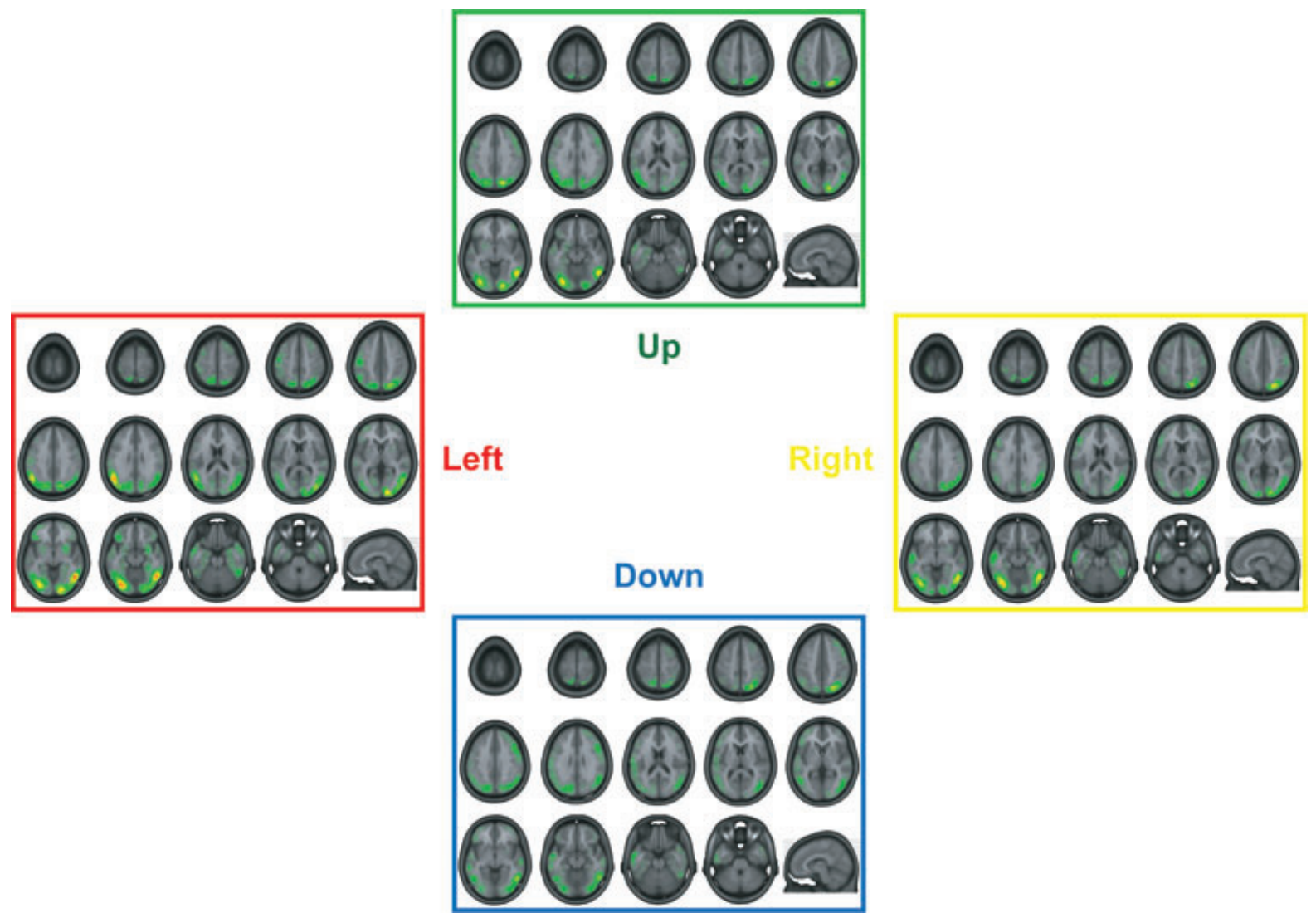

FIG. 4. 
Right vs Left
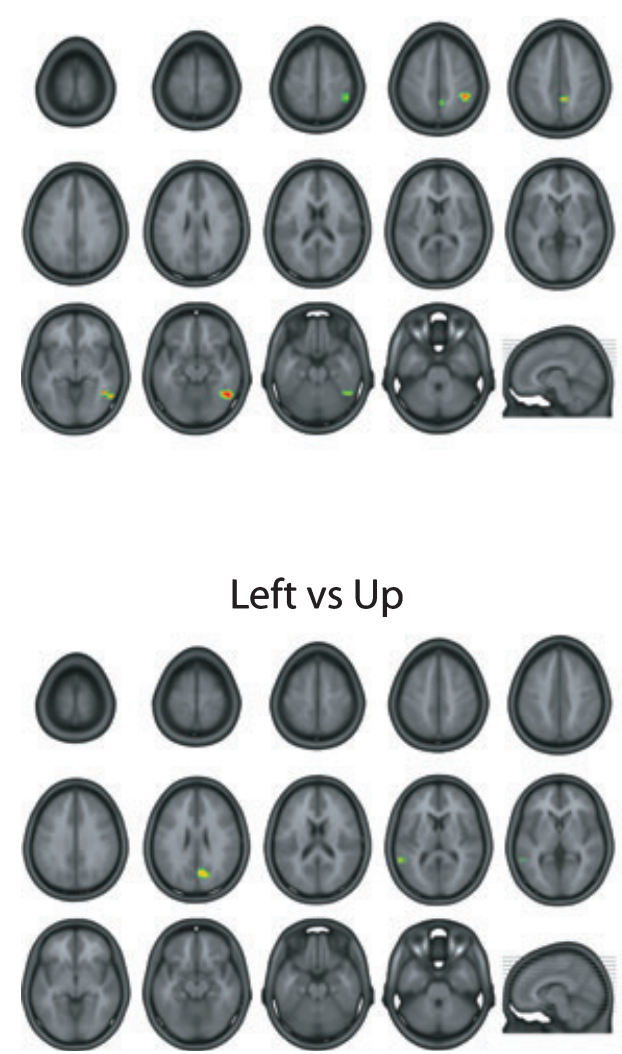

Right vs Down

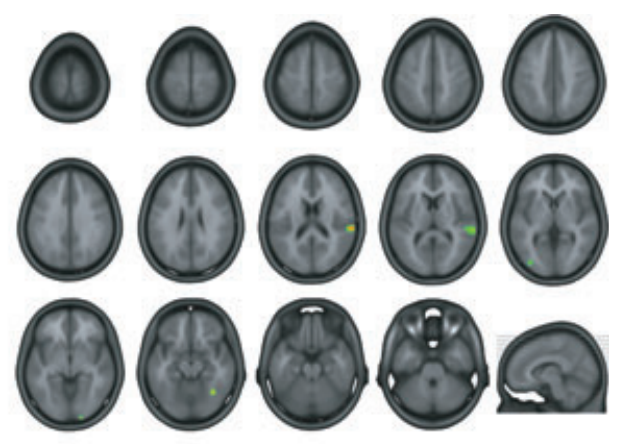

\section{Up vs Down}
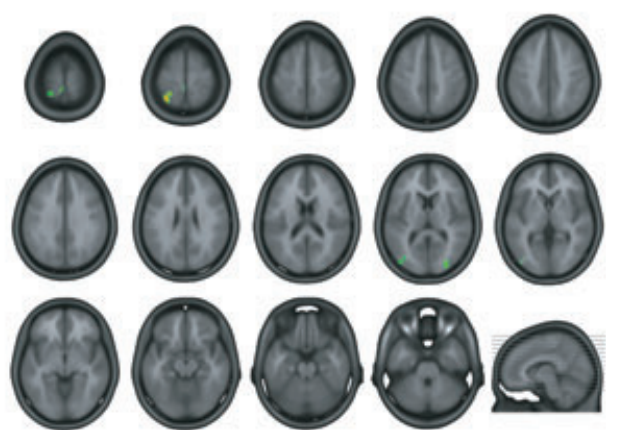

FIG. 5. Inverse solution: statistical comparisons between directed motion conditions. Statistical analyses of the inverse solutions (depicted at a significance level of $P<0.01)$ are shown for the following directional comparisons: right vs. left, right vs. down, left vs. up and up vs. down.

A

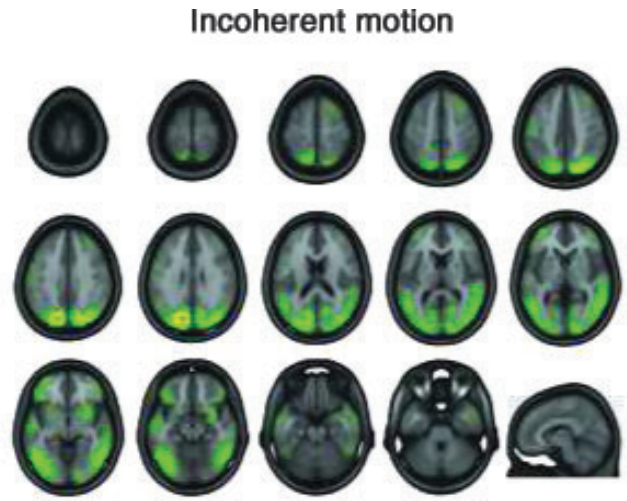

B

Incoherent motion vs. directed motion
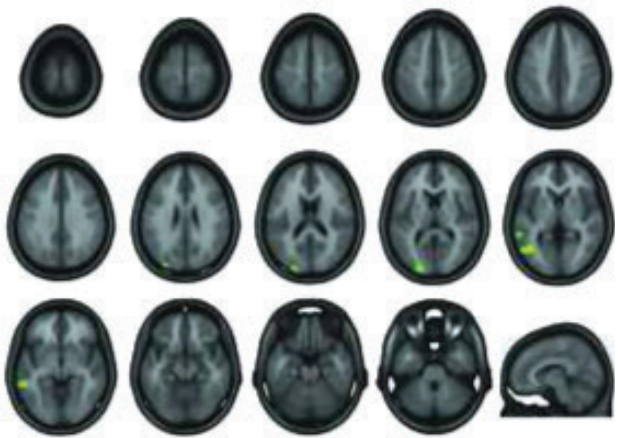

FIG. 6. Inverse solution: brain activations and statistical comparison of the incoherent motion condition with directed motion conditions. (A) The brain activation of the incoherent motion condition is localized in bilateral parieto-occipital and bilateral temporo-occipital cortex. (B) Statistical analyses of the inverse solutions (depicted at a significance level of $P<0.01$ ) are shown for the comparison of the undirected motion condition vs. the mean of the four directed motion conditions.

FIG. 3. Directed motion - incoherent motion differences revealed by ERP trace analysis and ERP mapping. (A) ERP traces corresponding to directed (in grey) and incoherent (in black) motion are plotted. Respectively from left to right are plotted the P120 component at Oz electrodes, the N170 component at Ot* and the P220 component at Cz. (B) Topographical clustering results show different scalp maps occurring at $170 \mathrm{~ms}$ for the directed and incoherent motion conditions (left part). Scalp maps corresponding to both segments are also depicted (middle). The segment in grey correlated with the directed motion condition, whereas the segment in black occurred in the incoherent motion condition. The graph on the right represents the mean duration of the grey and the black map across all subjects for the two conditions.

FIG. 4. Inverse solution: brain activations of each directed motion condition. Brain activations of the directed motion conditions are shown for right (yellow), left (red), up (green) and down (blue) directions. Brain activations are located in bilateral temporo-occipital, bilateral parieto-occipital and occipital areas (see text for further details). 
opposite direction to the one presented during adaptation). Previous studies also showed that the latency of the N170 component relates to motion parameters including velocity (Muller et al., 1998; Maruyama et al., 2002). Although we could not confirm an effect of motion direction on the latency of any of the evaluated ERP components (neither directional nor motion effects were observed in this study), our data on the amplitude of the N170 component corroborate previous findings and reveal that the N170 component is tuned to processing related to the detection and discrimination of motion direction.

The role of the P220 component in processing motion attributes of visual stimuli has been explored by only a small number of authors. Those studies suggested a modulation of the P220 component by stimulus velocity (Hoffmann \& Bach, 1997) and motion adaptation (Hoffmann et al., 1999), but also by motion complexity (Kuba et al., 2007). Extending these previous studies the present data show that the directional, but not the motion, effect also extends to the P220 component. The P120 component showed neither the directional nor the motion effect, compatible with observations showing that this earlier component reflects brain activity involved in encoding other visual stimulus attributes such as luminance, contrast, pattern reversal, as well as the onset and offset of a stimulus (Kubova \& Kuba, 1993; Bach \& Ullrich, 1997; Kuba et al., 2007), but not its direction.

Eye movement analysis revealed correct fixation and excluded saccadic eye movements that might have artefacted our EEG data during the appearance of the directional ERP components and the directional EP maps. We detected smooth pursuit eye movements (with very small amplitudes of $<0.1^{\circ}$ that started only at $250 \mathrm{~ms}$ after stimulus onset). As our visual stimuli extended $12 \times 12^{\circ}$ of visual angle and as these small eye movements were detected after directional ERP components and EP maps, we conclude that these directional effects are not eye movement artefacts. Nevertheless, we cannot exclude the possibility that the suppression or planning of eye movements may have influenced directional motion processing in the extrastriate cortex and some of the reported ERP findings, especially as both are co-localized in similar brain regions (Dieterich et al., 1998; Hoffmann \& Bach, 2002; Bense et al., 2006; Tikhonov et al., 2007). Our analysis excluded eye movements $>0.1^{\circ}$, but as a recent study by Yuval-Greenberg et al. (2008) reported that even small-amplitude saccades may lead to EEG oscillations and changes, we cannot exclude that small eye movements $\left(<0.1^{\circ}\right)$ may have modulated the EEG data reported here.

In summary, the present single-trace analysis reveals that the amplitudes of the N170 and P220 component encode differences in motion direction, whereas only the N170 component also encodes the presence or absence of directed motion (motion effect) without significant latency differences at both ERP components. Based on the data discussed so far it is not possible to determine definitively whether such directional ERP differences are due to differences in strength of brain activations of similar brain regions, due to the activation of different brain regions, or both. Moreover, these data do not distinguish whether stimulus processing leading to directional effects at the N170 and the P220 component reflect one single longer brain process encompassing the two ERP components, two separate shorter brain processes or several sequential shorter brain processes. This reasoning also applies to the motion effect at the N170 component. For this we now turn to map analysis and distributed source localization.

\section{Map analysis and electrical neuroimaging}

ERP mapping showed that the directional effect is due to the activation of distinct brain structures that encode the different motion directions.
Temporal cluster analysis showed that the directional effect was present during the entire time period from 164 to $226 \mathrm{~ms}$. Such a result constitutes evidence against the existence of two sequential processing steps (one for the N170 component and another for the P220 component). Instead, our analysis revealed one single and longer brain process for the processing of each motion direction. In addition, we found separate maps for each tested direction, suggesting that the brain encodes motion direction during this time window by distinct brain activations. What does this mean with respect to the underlying neural mechanisms involved in the processing of motion direction? Scalp topographies or ERP maps do not change randomly, but remain constant for a period of time in a certain configuration and then switch to a new stable configuration. Each distinct map is thought to represent a given 'functional microstate' of the brain or a given computational step during information processing (Lehmann et al., 1987; Michel et al., 2001, 2004). As our topographical analysis revealed the presence of maps that were specific for each tested motion direction (in the time period from 164 to $226 \mathrm{~ms}$ ), this suggests that each motion direction leads to the activation of a distinct distributed network. A given map represents the sum of all momentarily active neuronal populations (or sources). Accordingly, changes in map configuration may be due to relative changes in strength of only one (or more) of these sources with respect to other stable sources, or activation of distinct sources for the tested directions (Lehmann \& Skrandies, 1980; Lehmann et al., 1987; Michel et al., 1999; Katayama et al., 2007; Murray et al., 2008). Based on our inverse solution data, it cannot yet be decided which of these two mechanisms (or both) are present (see below for further discussion). Yet, this electrophysiological correlate of motion direction selectivity is compatible with previous data on motion direction representation in the human extrastriate cortex (Blanke et al., 2002, 2003). These previous clinical data showed that intracranial electrical stimulation (Blanke et al., 2002) and focal brain damage (Blanke et al., 2003) of the extrastriate cortex (including area MT+/V5) may lead to direction-selective motion blindness, compatible with macroscopic representation of motion direction in the human extrastriate cortex. Thus, these clinical data showed that focal extrastriate damage may lead to the loss or significant deficits in motion perception for one or two motion directions, while the perception of motion in other directions was unimpaired (Blanke et al., 2003).

The application of a linear inverse solution to each motion directionselective map (Fig. 4) and statistical analysis carried out at the level of the inverse solutions (Fig. 5) further extended these observations, and is compatible with results of previous EEG and MEG studies localizing the generator of extrastriate activity at $\sim 170 \mathrm{~ms}$ after stimulus onset (Probst et al., 1993; Nakamura \& Ohtsuka, 1999; Schellart et al., 2004) in occipito-temporal or occipito-parietal cortex (Probst et al., 1993; Nakamura \& Ohtsuka, 1999), but also at more posterior sites estimated in close proximity to area V3a (Nakamura \& Ohtsuka, 1999; Schellart et al., 2004). Using MEG and fMRI, Ahlfors et al. (1999) and Bundo et al. (2000) localized the N170 component even to a more extended network of brain areas including, in addition to the abovementioned areas, the posterior superior temporal cortex and frontal eyes field. Our data using a linear inverse solution are concordant with these results. We observed the activation of a common extrastriate network of brain regions for all tested motion directions consisting of temporo-occipital cortex bilaterally as well as parieto-occipital cortex bilaterally. Motion direction processing in temporo-occipital and parieto-occipital cortex has also been found in the abovementioned neurological studies that revealed two main lesion overlap regions that were associated with direction-selective motion blindness (Blanke et al., 2003), echoing the findings of the present electrophysiological study in healthy subjects. 
Activation of temporo-occipital cortex, as suggested by the present data, concords with the stereotaxic coordinates proposed for area $\mathrm{MT}+$ /V5 using positron emission tomography and fMRI (Watson et al., 1993; Dupont et al., 1994, 1997; de Jong et al., 1994; Tootell \& Taylor, 1995; Tootell et al., 1995; Goebel et al., 1998). Activations in parieto-occipital cortex were found in proximity with the stereotaxic coordinates proposed for area V3a (Braddick et al., 2000), but may also reflect motion processing in brain regions close to the posterior aspects of the intraparietal sulcus (Sunaert et al., 1999; Braddick et al., 2000).

Yet, the present data also reveal differential activations between the different motion directions: we report maximal activation for the different motion directions in occipito-temporal cortex for three directions (left, right, up) and for the down direction in parietooccipital cortex, and that these maximal activations varied with respect to activated hemisphere and the strength of activation of other nodes with the common network. We currently do not know whether these directional differences are due to amplitude changes of one or more nodes coding for one direction or whether these directional differences are due to the activation of distinct directional networks. Further electrophysiological work using intracranial recordings (i.e. Blanke et al., 1999, 2005) as well as work using fMRI or transcranial magnetic stimulation will be necessary to further elucidate the underlying functional neuroanatomy of such directional macroscopic mechanisms in the visual cortex. Because the different studies reviewed here and the present study were carried out in different species with different visual stimuli, and using different neuroimaging and electrophysiological methods, further caution seems necessary. Collectively, these clinical, electrophysiological and neuroimaging data suggest that motion direction may be represented in the human cortex not only at the microscopic columnar level (Albright et al., 1984; Diogo et al., 2002), but also at the macroscopic level and extending beyond $\mathrm{MT}+/ \mathrm{V} 5$ in temporo-occipital and parieto-occipital areas in the extrastriate cortex. Importantly, we found that only brain activity between 164 and $226 \mathrm{~ms}$ in these regions is tuned for visual motion direction processing, and that such electrophysiological activity related to directional processing overlaps only initially with processing related to incoherent motion perception.

\section{Acknowledgements}

This study was supported by grants from the Swiss National Science Foundation (\#3100-067874.02 to M.M and O.B., \#310000-114008 and \#3200B0-104100 to S.S.). Analyses were carried out using Cartool ${ }^{\odot}$. The Cartool software is developed by Denis Brunet and supported by the CIBM. Visual stimuli were created using Cogent Graphics developed by John Romaya (Wellcome Department of Imaging Neuroscience; London, UK). We also thank Anna Brooks and the two anonymous reviewers for helpful comments on an earlier version of this article, and Marc Repnow for expert help with the eye movement recording.

\section{Abbreviations}

EEG, electroencephalography; EOG, electroocculography; ERP, event-related potential; fMRI, functional magnetic resonance imaging; GEV, global explained variance; LAURA, local auto-regressive average; MEG, magnetoencephalography; MT, medial temporal area; RDK, random dot kinematogram; V3a, V3 accessory area.

\section{References}

Ahlfors, S.P., Simpson, G.V., Dale, A.M., Belliveau, J.W., Liu, A.K., Korvenoja, A., Virtanen, J., Huotilainen, M., Tootell, R.B., Aronen, H.J. \& Ilmoniemi, R.J. (1999) Spatiotemporal activity of a cortical network for processing visual motion revealed by MEG and fMRI. J. Neurophysiol., 82 $2545-2555$.

Albright, T.D. \& Stoner, G.R. (1995) Visual motion perception. Proc. Natl. Acad. Sci. U.S.A., 92, 2433-2440.

Albright, T.D., Desimone, R. \& Gross, C.G. (1984) Columnar organization of directionally selective cells in visual area MT of the macaque. J. Neurophysiol., 51, 16-31.

Arzy, S., Thut, G., Mohr, C., Michel, C.M. \& Blanke, O. (2006) Neural basis of embodiment: distinct contributions of temporoparietal junction and extrastriate body area. $J$. Neurosci., 26, 8074-8081.

Bach, M. \& Hoffmann, M.B. (2000) Visual motion detection in man is governed by non-retinal mechanisms. Vision Res., 40, 2379-2385.

Bach, M. \& Ullrich, D. (1994) Motion adaptation governs the shape of motionevoked cortical potentials. Vision Res., 34, 1541-1547.

Bach, M. \& Ullrich, D. (1997) Contrast dependency of motion-onset and pattern-reversal VEPs: interaction of stimulus type, recording site and response component. Vision Res., 37, 1845-1849.

Bense, S., Janusch, B., Schlindwein, P., Bauermann, T., Vucurevic, G., Brandt, T., Stoeter, P. \& Dieterich, M. (2006) Direction-dependent visual cortex activation during horizontal optokinetic stimulation (fMRI study). Hum. Brain Mapp., 27, 296-305.

Blanke, O., Morand, S., Thut, G., Michel, C.M., Spinelli, L., Landis, T. \& Seeck, M. (1999) Visual activity in the human frontal eye field. Neuroreport, 10, 925-930.

Blanke, O., Landis, T., Safran, A.B. \& Seeck, M. (2002) Direction-specific motion blindness induced by focal stimulation of human extrastriate cortex. Eur. J. Neurosci., 15, 2043-2048.

Blanke, O., Landis, T., Mermoud, C., Spinelli, L. \& Safran, A.B. (2003) Direction-selective motion blindness after unilateral posterior brain damage. Eur. J. Neurosci., 18, 709-722.

Blanke, O., Mohr, C., Michel, C.M., Pascual-Leone, A., Brugger, P., Seeck, M., Landis, T. \& Thut, G. (2005) Linking out-of-body experience and self processing to mental own-body imagery at the temporoparietal junction. $J$. Neurosci., 25, 550-557.

Blanke, O., Brooks, A., Mercier, M., Spinelli, L., Adriani, M., Lavanchy, L., Safran, A.B. \& Landis, T. (2007) Distinct mechanisms of form-frommotion perception in human extrastriate cortex. Neuropsychologia, 45, 644-653.

Braddick, O.J., O’Brien, J.M., Wattam-Bell, J., Atkinson, J. \& Turner, R. (2000) Form and motion coherence activate independent, but not dorsal/ventral segregated, networks in the human brain. Curr. Biol., 10, 731-734.

Bundo, M., Kaneoke, Y., Inao, S., Yoshida, J., Nakamura, A. \& Kakigi, R. (2000) Human visual motion areas determined individually by magnetoencephalography and 3D magnetic resonance imaging. Hum. Brain Mapp., 11, 33-45.

Culham, J., He, S., Dukelow, S. \& Verstraten, F.A. (2001) Visual motion and the human brain: what has neuroimaging told us? Acta Psychol. (Amst), 107, 69-94.

Dieterich, M., Bucher, S.F., Seelos, K.C. \& Brandt, T. (1998) Horizontal or vertical optokinetic stimulation activates visual motion-sensitive, ocular motor and vestibular cortex areas with right hemispheric dominance. An fMRI study. Brain, 121(Pt 8), 1479-1495.

Diogo, A.C., Soares, J.G., Albright, T.D. \& Gattass, R. (2002) Twodimensional map of direction selectivity in cortical visual area MT of Cebus monkey. An. Acad. Bras. Cienc., 74, 463-476.

Dumoulin, S.O., Bittar, R.G., Kabani, N.J., Baker, C.L. Jr, Le Goualher, G., Bruce Pike, G. \& Evans, A.C. (2000) A new anatomical landmark for reliable identification of human area V5/MT: a quantitative analysis of sulcal patterning. Cereb. Cortex, 10, 454-463.

Dupont, P., Orban, G.A., De Bruyn, B., Verbruggen, A. \& Mortelmans, L. (1994) Many areas in the human brain respond to visual motion. J. Neurophysiol., 72, 1420-1424.

Dupont, P., De Bruyn, B., Vandenberghe, R., Rosier, A.M., Michiels, J., Marchal, G., Mortelmans, L. \& Orban, G.A. (1997) The kinetic occipital region in human visual cortex. Cereb. Cortex, 7, 283-292.

Ffytche, D.H., Guy, C.N. \& Zeki, S. (1995) The parallel visual motion inputs into areas V1 and V5 of human cerebral cortex. Brain, 118(Pt 6), 13751394.

Goebel, R., Khorram-Sefat, D., Muckli, L., Hacker, H. \& Singer, W. (1998) The constructive nature of vision: direct evidence from functional magnetic resonance imaging studies of apparent motion and motion imagery. Eur. $J$. Neurosci., 10, 1563-1573.

Grave de Peralta Menendez, R., Gonzalez Andino, S., Lantz, G., Michel, C.M. \& Landis, T. (2001) Noninvasive localization of electromagnetic epileptic activity. I. Method descriptions and simulations. Brain Topogr., 14, 131-137. 
Grave de Peralta Menendez, R., Murray, M.M., Michel, C.M., Martuzzi, R. \& Gonzalez Andino, S.L. (2004) Electrical neuroimaging based on biophysical constraints. Neuroimage, 21, 527-539.

Grave de Peralta-Menendez, R. \& Gonzalez-Andino, S.L. (1998) A critical analysis of linear inverse solutions to the neuroelectromagnetic inverse problem. IEEE Trans. Biomed. Eng., 45, 440-448.

Heinrich, S.P., van der Smagt, M.J., Bach, M. \& Hoffmann, M.B. (2004) Electrophysiological evidence for independent speed channels in human motion processing. J. Vis., 4, 469-475.

Heinrich, S.P., Renkl, A.E. \& Bach, M. (2005) Pattern specificity of human visual motion processing. Vision Res., 45, 2137-2143.

Hoffmann, M. \& Bach, M. (1997) Motion onset VEPs: topographic distribution and dependence on stimulus velocity reveals two components. Brain Topogr., 10, 171.

Hoffmann, M.B. \& Bach, M. (2002) The distinction between eye and object motion is reflected by the motion-onset visual evoked potential. Exp. Brain Res., 144, 141-151.

Hoffmann, M., Dorn, T.J. \& Bach, M. (1999) Time course of motion adaptation: motion-onset visual evoked potentials and subjective estimates. Vision Res., 39, 437-444.

Hoffmann, M.B., Unsold, A.S. \& Bach, M. (2001) Directional tuning of human motion adaptation as reflected by the motion VEP. Vision Res., 41, 2187-2194.

de Jong, B.M., Shipp, S., Skidmore, B., Frackowiak, R.S. \& Zeki, S. (1994) The cerebral activity related to the visual perception of forward motion in depth. Brain, 117(Pt 5), 1039-1054.

Katayama, H., Gianotti, L.R., Isotani, T., Faber, P.L., Sasada, K., Kinoshita, T. \& Lehmann, D. (2007) Classes of multichannel EEG microstates in light and deep hypnotic conditions. Brain Topogr, 20, 7-14.

Kuba, M., Kubova, Z., Kremlacek, J. \& Langrova, J. (2007) Motion-onset VEPs: characteristics, methods, and diagnostic use. Vision Res., 47, 189-202.

Kubova, Z. \& Kuba, M. (1993) Visual evoked potentials in reversal and motion-onset stimulation of visual fields in patients with retrobulbar neuritis. Cesk. Oftalmol., 49, 278-285.

Lehmann, D. \& Skrandies, W. (1980) Reference-free identification of components of checkerboard-evoked multichannel potential fields. Electroencephalogr. Clin. Neurophysiol., 48, 609-621.

Lehmann, D., Ozaki, H. \& Pal, I. (1987) EEG alpha map series: brain microstates by space-oriented adaptive segmentation. Electroencephalogr. Clin. Neurophysiol., 67, 271-288.

Maruyama, K., Kaneoke, Y., Watanabe, K. \& Kakigi, R. (2002) Human cortical responses to coherent and incoherent motion as measured by magnetoencephalography. Neurosci. Res., 44, 195-205.

Maurer, J.P. \& Bach, M. (2003) Isolating motion responses in visual evoked potentials by preadapting flicker-sensitive mechanisms. Exp. Brain Res., 151, 536-541.

Michel, C.M., Grave de Peralta, R., Lantz, G., Gonzalez Andino, S., Spinelli, L., Blanke, O., Landis, T. \& Seeck, M. (1999) Spatiotemporal EEG analysis and distributed source estimation in presurgical epilepsy evaluation. J. Clin. Neurophysiol., 16, 239-266.

Michel, C.M., Thut, G., Morand, S., Khateb, A., Pegna, A.J., Grave de Peralta, R., Gonzalez, S., Seeck, M. \& Landis, T. (2001) Electric source imaging of human brain functions. Brain Res. Brain Res. Rev., 36, 108-118.

Michel, C.M., Seeck, M. \& Murray, M.M. (2004) The speed of visual cognition. Suppl. Clin. Neurophysiol., 57, 617-627.
Muller, R., Gopfert, E., Breuer, D. \& Greenlee, M.W. (1998) Motion VEPs with simultaneous measurement of perceived velocity. Doc. Ophthalmol., 97, 121-134.

Murray, M.M., Michel, C.M., Grave de Peralta, R., Ortigue, S., Brunet, D., Gonzalez Andino, S. \& Schnider, A. (2004) Rapid discrimination of visual and multisensory memories revealed by electrical neuroimaging. Neuroimage, 21, 125-135.

Murray, M.M., Brunet, D. \& Michel, C.M. (2008) Topographic ERP analyses: a step-by-step tutorial review. Brain Topogr., 20, 249-264.

Nakamura, Y. \& Ohtsuka, K. (1999) Topographical analysis of motiontriggered visual-evoked potentials in man. Jpn. J. Ophthalmol., 43, 3643.

Nakamura, H., Kashii, S., Nagamine, T., Matsui, Y., Hashimoto, T., Honda, Y, \& Shibasaki, H. (2003) Human V5 demonstrated by magnetoencephalography using random dot kinematograms of different coherence levels. Neurosci. Res., 46, 423-433.

Niedeggen, M. \& Wist, E.R. (1998) Motion evoked brain potentials parallel the consistency of coherent motion perception in humans. Neurosci. Lett., 246, 61-64.

Niedeggen, M. \& Wist, E.R. (1999) Characteristics of visual evoked potentials generated by motion coherence onset. Brain Res. Cogn. Brain Res., 8, 95105.

Pascual-Marqui, R.D., Michel, C.M. \& Lehmann, D. (1995) Segmentation of brain electrical activity into microstates: model estimation and validation. IEEE Trans. Biomed. Eng., 42, 658-665.

Probst, T., Plendl, H., Paulus, W., Wist, E.R. \& Scherg, M. (1993) Identification of the visual motion area (area V5) in the human brain by dipole source analysis. Exp. Brain Res., 93, 345-351.

Schellart, N.A., Trindade, M.J., Reits, D., Verbunt, J.P. \& Spekreijse, H. (2004) Temporal and spatial congruence of components of motion-onset evoked responses investigated by whole-head magneto-electroencephalography. Vision Res., 44, 119-134.

Sunaert, S., Van Hecke, P., Marchal, G. \& Orban, G.A. (1999) Motion-responsive regions of the human brain. Exp. Brain Res., 127, 355370 .

Thierry, G., Martin, C.D., Downing, P. \& Pegna, A.J. (2007) Controlling for interstimulus perceptual variance abolishes N170 face selectivity. Nat. Neurosci., 10, 505-511.

Tikhonov, A., Handel, B., Haarmeier, T., Lutzenberger, W. \& Thier, P. (2007) Gamma oscillations underlying the visual motion aftereffect. Neuroimage, 38, 708-719.

Tootell, R.B. \& Taylor, J.B. (1995) Anatomical evidence for MT and additional cortical visual areas in humans. Cereb. Cortex, 5, 39-55.

Tootell, R.B., Reppas, J.B., Kwong, K.K., Malach, R., Born, R.T., Brady, T.J., Rosen, B.R. \& Belliveau, J.W. (1995) Functional analysis of human MT and related visual cortical areas using magnetic resonance imaging. J. Neurosci., 15, 3215-3230.

Watson, J.D., Myers, R., Frackowiak, R.S., Hajnal, J.V., Woods, R.P., Mazziotta, J.C., Shipp, S. \& Zeki, S. (1993) Area V5 of the human brain: evidence from a combined study using positron emission tomography and magnetic resonance imaging. Cereb. Cortex, 3, 79-94.

Yuval-Greenberg, S., Tomer, O., Keren, A.S., Nelken, I. \& Deouell, L.Y. (2008) Transient induced gamma-band response in EEG as a manifestation of miniature saccades. Neuron, 58, 429-441. 